\title{
The nicotinic acetylcholine receptor gene family of the silkworm, Bombyx mori
}

\author{
Ya-Ming Shao', Ke Dong² and Chuan-Xi Zhang*1
}

Address: ${ }^{1}$ Institute of Insect Science, Zhejiang University, Hangzhou, Zhejiang, 310029, China and ${ }^{2}$ Department of Entomology and Neuroscience Program, Michigan State University, East Lansing, MI 48824, USA

Email: Ya-Ming Shao - symhhit@sohu.com; Ke Dong - dongk@msu.edu; Chuan-Xi Zhang* - chxzhang@zju.edu.cn

* Corresponding author

Published: 15 September 2007

BMC Genomics 2007, 8:324 doi:10.1/86/147|-2164-8-324
Received: 8 August 2007

Accepted: 15 September 2007

This article is available from: http://www.biomedcentral.com/I47I-2/64/8/324

(c) 2007 Shao et al; licensee BioMed Central Ltd.

This is an Open Access article distributed under the terms of the Creative Commons Attribution License (http://creativecommons.org/licenses/by/2.0), which permits unrestricted use, distribution, and reproduction in any medium, provided the original work is properly cited.

\begin{abstract}
Background: Nicotinic acetylcholine receptors (nAChRs) mediate fast synaptic cholinergic transmission in the insect central nervous system. The insect $n A C h R$ is the molecular target of a class of insecticides, neonicotinoids. Like mammalian nAChRs, insect nAChRs are considered to be made up of five subunits, coded by homologous genes belonging to the same family. The $n A C h R$ subunit genes of Drosophila melanogaster, Apis mellifera and Anopheles gambiae have been cloned previously based on their genome sequences. The silkworm Bombyx mori is a model insect of Lepidoptera, among which are many agricultural pests. Identification and characterization of B. mori $\mathrm{nAChR}$ genes could provide valuable basic information for this important family of receptor genes and for the study of the molecular mechanisms of neonicotinoid action and resistance.

Results: We searched the genome sequence database of $B$. mori with the fruit fly and honeybee $\mathrm{nAChRs}$ by tBlastn and cloned all putative silkworm nAChR cDNAs by reverse transcriptasepolymerase chain reaction (RT-PCR) and rapid amplification of CDNA ends (RACE) methods. $B$. mori appears to have the largest known insect $n A C h R$ gene family to date, including nine $\alpha$-type subunits and three $\beta$-type subunits. The silkworm possesses three genes having low identity with others, including one $\alpha$ and two $\beta$ subunits, $\alpha 9, \beta 2$ and $\beta 3$. Like the fruit fly and honeybee counterparts, silkworm nAChR gene $\alpha 6$ has RNA-editing sites, and $\alpha 4, \alpha 6$ and $\alpha 8$ undergo alternative splicing. In particular, alternative exon 7 of $\mathrm{Bm \alpha} 8$ may have arisen from a recent duplication event. Truncated transcripts were found for $\mathrm{Bm \alpha} 4$ and $\mathrm{Bm \alpha 5}$.

Conclusion: B. mori possesses a largest known insect $n A C h R$ gene family characterized to date, including nine $\alpha$-type subunits and three $\beta$-type subunits. RNA-editing, alternative splicing and truncated transcripts were found in several subunit genes, which might enhance the diversity of the gene family.
\end{abstract}

\section{Background}

The nicotinic acetylcholine receptor (nAChR) is a ligandgated ion channel (LGIC) that mediates fast synaptic cholinergic transmission in the insect central nervous system [1]. A nAChR is formed by five subunits arranged around a central pore that is permeable to cations [2]. These subunits are encoded by multiple $\alpha$ - and $\beta$ subunit genes. Diverse nAChRs, differing in subunit composition, have different electrophysiological and pharmacological profiles [3]. Agonists, such as acetylcholine, bind to the $\mathrm{N}$ - 
terminal extracellular domain of the subunits at a site formed by six loops (Loop A-F) located at subunit interfaces. The $\mathrm{N}$-terminal extracellular domain also contains a Cys-loop, characteristic of cys-Loop LGICs, which consists of two disulphide-bond forming cysteines separated by 13 residues [2]. Each subunit also has four transmembrane regions (TM1-TM4). The subunits with two vicinal Cys at loop $\mathrm{C}$ which are involved in acetylcholine binding are classified into $\alpha$ type, whereas other subunits lacking the two Cys residues are regarded as non- $\alpha$ type [4]. Phosphorylation sites are found in a long intracellular loop between TM3 and TM4, which may modulate the receptor activity $[5,6]$.

The genomes of Drosophila melanogaster, Anopheles gambiae, Apis mellifera have been completely sequenced and their nAChR gene families have been annotated. D. melanogaster has seven $\alpha$ and three $\beta$ subunits [7], A. gambiae has nine $\alpha$ and one $\beta$ [8], and $A$. mellifera possesses nine $\alpha$ and two $\beta$ [9]. Mammals and chicken have 16 and 17 nAChR subunits respectively [10]. Compared with vertebrates, insects seem to have a small nAChR gene family. However, recent studies showed that insects may enhance the functional diversity through alternative splicing of exons and RNA editing of transcripts [7,9]. In Drosophila, alternative splicing leads to the loss of some exons or retention of introns in mature nAChR mRNA [7].

Although study of insect nAChRs is still at an early stage, researchers have exploited $\mathrm{nAChR}$ as an insecticide target for a long time [11]. To defend against insects and other herbivores, plants synthesize compounds having insecticidal activities. Nicotine extracted from tobacco, for example, has been used as a commercial insecticide extensively, which is a potent agonist of nAChR [11]. Neonicotinoids are a group of insecticides, including imidacloprid, thiamethoxam [12]. These insecticides exhibit low effective dosage, selective toxicity for insects over vertebrates, wide insecticidal activity, and excellent uptake in plants [13]. Since the first neonicotinoid insecticide imidacloprid was put into market, it has gained the fastest growing sales of any insecticide worldwide [12].

The silkworm, Bombyx mori, producing silk, is an important beneficial insect in many countries. Recently this insect gained more attention because the silkworm bodies can express foreign proteins after being infected with recombinant baculovirus [14]. B. mori is also a good model organism because of its large body size, ease of rearing [15], and the completion of the whole genome sequence $[16,17]$. B. mori belongs to Lepidoptera, the second largest order of Insecta, possessing about 200 thousand species. Most insects of this order are herbivores and eat plants at the larval stage and many are agricultural pests except for the silkworm. Up to date, five $\alpha$ and one $\beta$ subunits from Heliothis virescens, one $\alpha$ and one $\beta$ from Manduca sexta, one $\alpha$ from Chilo suppressalis and one $\beta$ subunit from Spodoptera exigua are deposited in GenBank. In addition, three alternative transcripts of $B$. mori $\alpha 6$ were reported [18]. Despite the agricultural importance of lepidopteran insects, the nAChR subunit gene family from this order remains unclear. In this report, we report the molecular characterization of the nAChR gene family from the silkworm.

\section{Results \\ Twelve candidate $n A C h R$ subunit genes in the B. mori genome}

We searched the $B$. mori genome sequence database for nAChR subunit genes by tBlastn [19]. Twelve candidate genes were identified. Through RT-PCR and RACE analyses, expression of all $12 \mathrm{nAChR}$ subunit genes was confirmed and complete open reading frames (ORFs) were obtained except Bma7. A 121 bp sequence encoding the signal peptide at the $\mathrm{N}$-terminus of $\mathrm{Bm} \alpha 7$ was obtained from an EST sequence [GenBank: BB982760] by BLAST search [20] and the complete ORF of $B m \alpha 7$ was then assembled. Alignment of predicted protein sequences (Figure 1) shows that all these subunits possess two Cys separated by 13 residues, typical of LGIC subunits, as well as six conserved Loops (Loops A-F) located at the N-terminal domain. Nine of them, having two vicinal Cys at Loop C, belong to type $\alpha$, whereas the other three lack this motif and are therefore of type $\beta$. All subunits carry a predicted signal peptide at the N-terminus, and all but one (Bma5) possess four transmembrane motifs (TM1-TM4) at the Cterminal domain, which are involved in forming an ion channel. Bma5 has only three transmembrane regions (TM1-TM3). Loops between TM3 and TM4 were highly variable and have predicted phosphorylation sites. Putative $\mathrm{N}$-glycosylation sites are present in the N-terminal extracellular domains of all nAChR subunits [21].

Most B. mori nAChRs show high levels of sequence identities with the counterparts of D. melanogaster, up to $86 \%$ (Table 1). However, Bmo5 shares a low sequence identity with D. melanogaster $\alpha 5$, although it shows the $48 \%$ amino acid identity with $A$. mellifera $\alpha 5$. We constructed a phylogenetic tree comprising 39 insect nAChR subunit protein sequences (Figure 2). In fruit fly, mosquito and honeybee, nAChR subunits $\alpha 1, \alpha 2, \alpha 3, \alpha 4, \alpha 6, \alpha 8$, and $\beta 1$ have strong corresponding relationships. Silkworm also has counterparts of these subunits, which are consequently named according to the existing nomenclature. However, subunits Bmo5 and Bmo7 are difficult to identify. In A. mellifera, $\alpha 7$ was named because its $\mathrm{N}$-terminal domain shared more identity with $D$. melanogaster $\alpha 7$ and another was named $\alpha 5$ [9]. We named B. mori $\alpha 5$ and $\alpha 7$ following the nomenclature of $A$. mellifera because they had strong corresponding relationships. D. melanogaster 


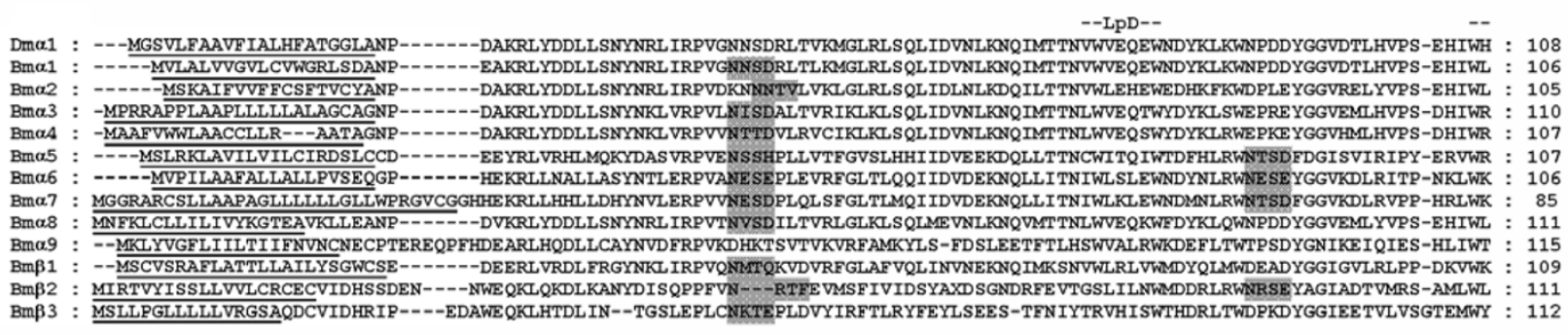

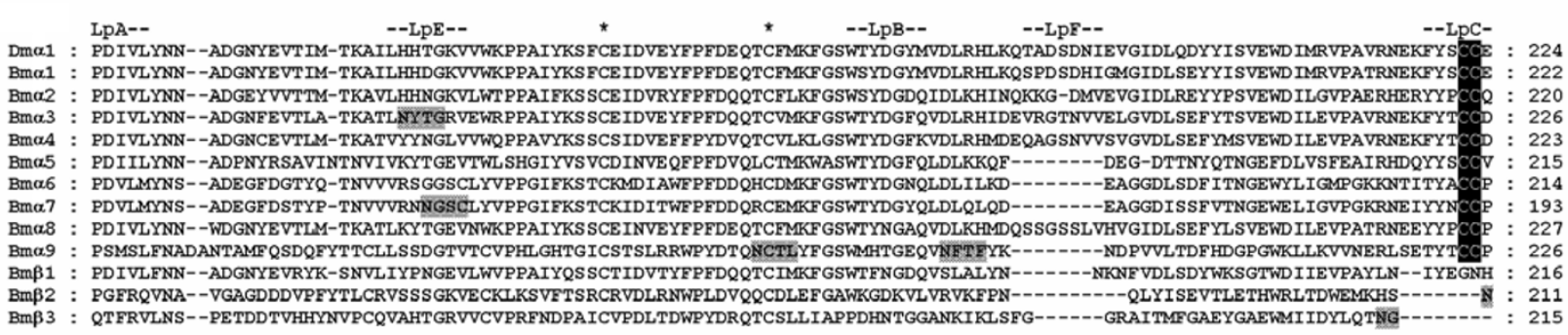

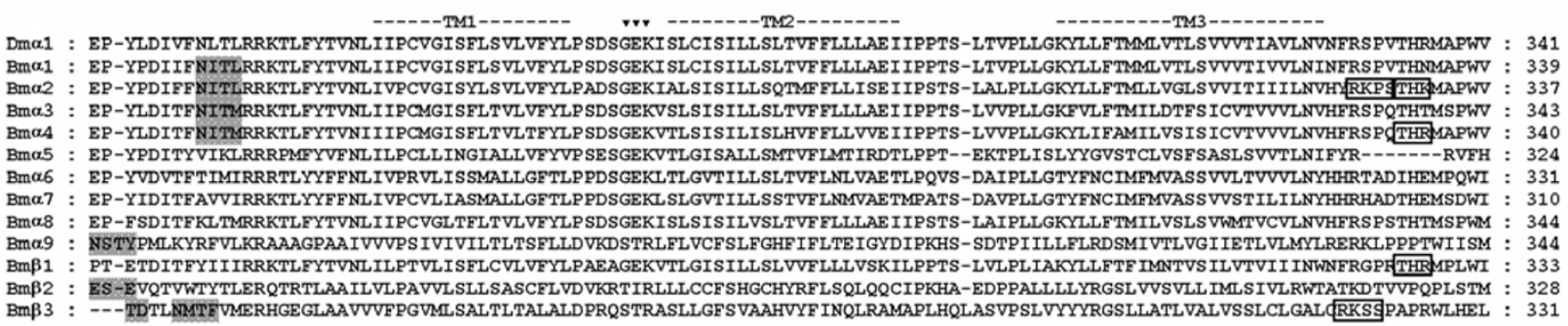

\begin{tabular}{|c|c|c|}
\hline & & \\
\hline 0,2 & KFFITKLPKLLLMRVPKDLLRDLAAQKIAGRSMMR---- & 433 \\
\hline $\mathrm{m} \alpha 3$ & 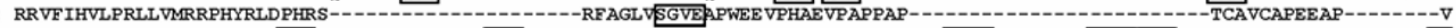 & 408 \\
\hline 04 & 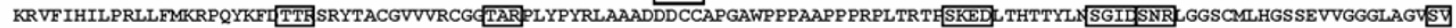 & 459 \\
\hline$n \alpha 5$ & 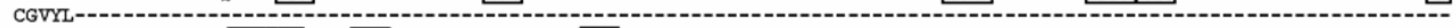 & 329 \\
\hline na6 & 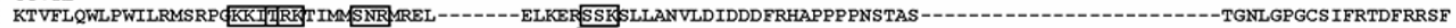 & 418 \\
\hline $\operatorname{Bma7}$ & 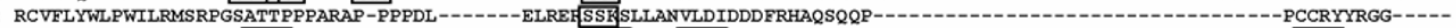 & 382 \\
\hline . & 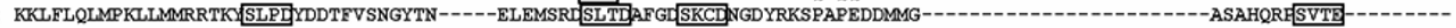 & 425 \\
\hline & RLVSNSPGKYVVFT-- & 359 \\
\hline & MELSDLHHPNCKINRAAGGG & 425 \\
\hline & AVMCSRWKML & \\
\hline
\end{tabular}

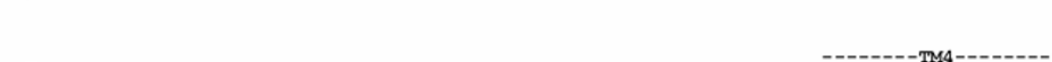

Dm $\alpha 1$ : AAAAAAAADLSPT--F-EKPYAREMEKTIEGSRFIAQHVKNKDKFESVEEDWKYVAMVLDRMFLWIFAIACVVGTALIILQAPSLHDQSQPIDILYSKIAKKKFELLKMGSENTL---- : 567 Bma1 : SPCRLDLDDGSPS--L-EKPYVREMEKTIEGSRFIAQHVKNKDKFSSVEDPWKYVAMVLDRIFLFLFTIACVLGTALIIFRAPTFYDNTKPIDILYSKIAKKKLELLRMGSEGDPGL-- : 533

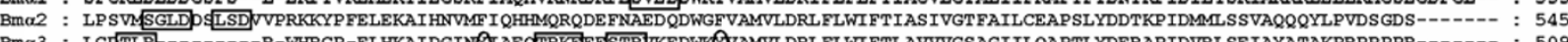

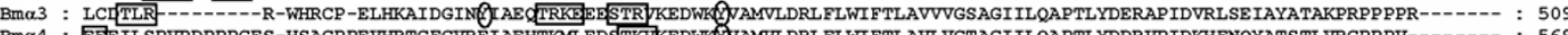
BmaA : E] 2 ILSPVPDPPGF S-HSACP PEVHRTCFCVRF IAEHTKMLED STKVKEDWKGVAMVLDRLFLWIFTLAVLVGTAGIILQAPTLYDDRVPIDKHFNQYATS TLVRCPPPV-------- : 569 Bma5 : - VR Bma7 : Bma8 : SEMML------RHLSP-EVAAALISVR:IAQHIKDADKDNEVIEDWKFMSMVLDRFFLWLFTIACFVGTFGIIFQSPSLYDTRVPVDQQISSIPMKKNNFFYPKDIETIGIIS : 532

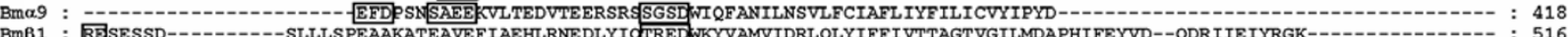

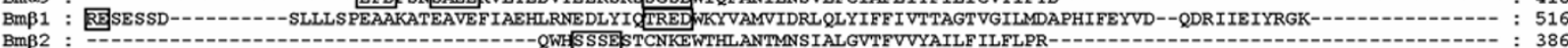

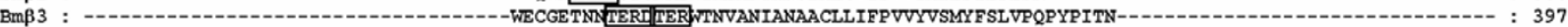

\section{Figure I}

Alignment of B. mori nAChR subunit protein sequences. D. melanogaster $\alpha$ l is included for comparison. $N$-terminal signal peptides are underlined. Position of loops (LPA-F) involved in ligand binding and transmembrane motifs (TMI-4) forming the ion channel are indicated. Sites of Cys residues involved in Cys-loop are marked with asterisks and the vicinal Cys residues characteristic of $\alpha$-type are showed in white letters. GEK motif associated with cation selectivity is indicated by inverted triangles. Putative $\mathrm{N}$-glycosylation sites are highlighted in shade. Potential PKA, PKC, and CK2 phosphorylation sites are boxed and potential tyrosine kinase phosphorylation sites are enclosed in ovals. 


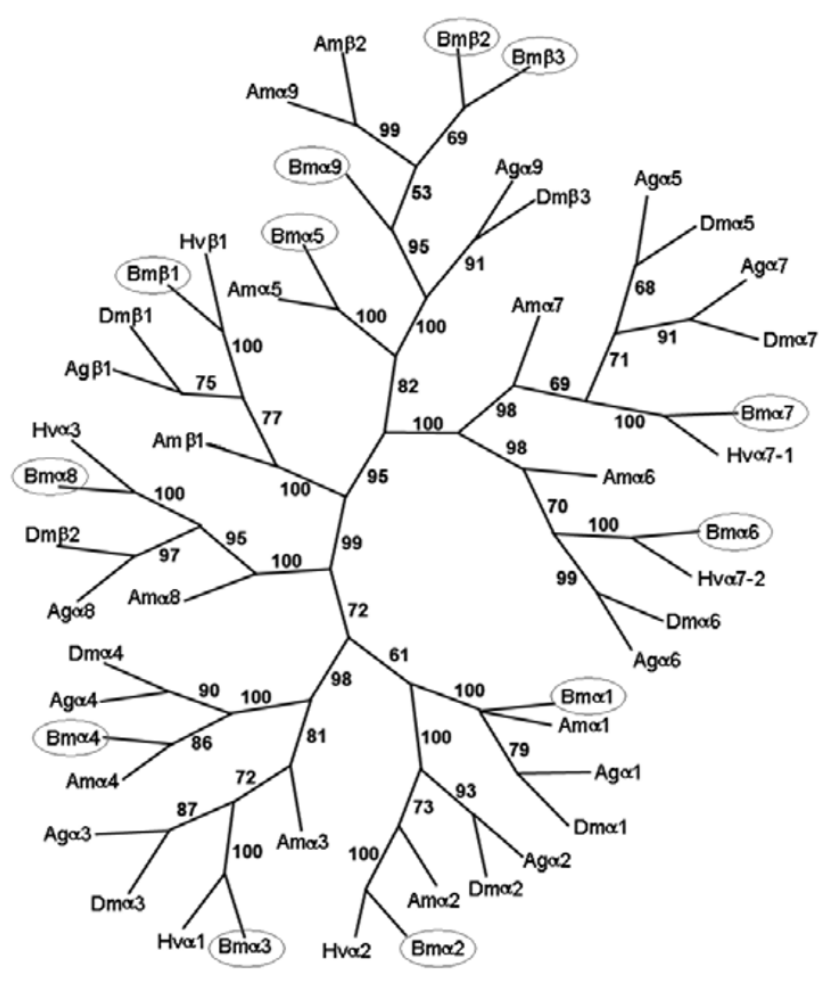

Figure 2

Phylogenetic relationship of insect $\mathbf{n A C h R}$ subunits.

The bootstrap $50 \%$ majority-rule consensus trees were made with the maximum parsimony method (PAUP, Version 4.0) using multiple alignments of amino acid sequences. Statistical support (percentage) for each node was evaluated by bootstrap analysis with I,000 replicates. B. mori $\mathrm{nAChRs}$ cloned by us are enclosed by ovals. The $n A C h R$ subunits shown in the tree are as follows including GenBank accession num-

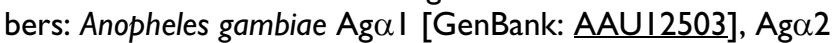

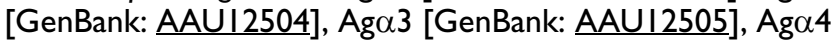

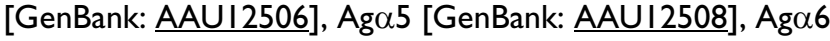
[GenBank: AAUI2509], Ag 27 [GenBank: AAUI25II], Ag $\alpha 8$ [GenBank: AAUI25I2], Ag $\alpha 9$ [GenBank: AAUI25I3], Ag $\beta$ [GenBank: AAUI25I4]. Drosophila melanogaster Dmal [GenBank: CAA30172], Dma2 [GenBank: CAA365I7], Dm 33 [GenBank: CAA75688], Dma4 [GenBank: CAB77445], Dma5 [GenBank: AAMI3390], Dma6 [GenBank: AAMI3392], Dma7 [GenBank: ABO26063], Dm $\beta$ I [GenBank: CAA2764I], Dm $\beta 2$ [GenBank: AAF56304], Dm $\beta 3$ [GenBank: CAC48166]. Apis mellifera Amal [GenBank: AAY87890], Ama2 [GenBank: AAS48080], Ama3 [GenBank: AAY8789I], Ama4 [GenBank: AAY87893], Am $\alpha 5$ [GenBank: AAS7578I], Am $\alpha 6$ [GenBank: AAY87895], Ama7 [GenBank: AAR92109], Amo8 [GenBank: AAM51823], Ama9 [GenBank: AAY87896], Am $\beta$ I [GenBank: AAY87897], Am $\beta 2$ [GenBank: AAY87898]. Heliothis virescens Hval [GenBank: AJ000399], Hva2 [GenBank: AF096878], Hvo3 [GenBank: AF096879], Hva7-I [GenBank: AFI43846], Hva7-2 [GenBank: AFI43847], Hv $\beta$ I [GenBank: AF096880]. $\beta 3$ and $A$. gambiae $\alpha 9$ had low amino acid identities with other gene family members. Recently, A. mellifera $\beta 2$ and $\alpha 9$ were also found having low sequence identities with other members of the nAChR family, and the two genes are located closely in the genome at a distance of $10 \mathrm{~kb}$. It was suggested that the two genes arose from a recent evolutionary duplication event [9]. As shown in Table 1, three B. mori subunits, $B m \alpha 9, B m \beta 2$, and $B m \beta 3$, have low sequence similarities with other $\mathrm{nAChR}$ subunits. Phylogenetic analysis indicates that $\mathrm{Bm} \beta 2$ and $\mathrm{Bm} \beta 3$ are clustered into one branch, suggesting that $\mathrm{Bm} \alpha 9$ had diverged before the two $\beta$ subunits separated evolutionarily. Bm $\alpha$ 9 shows $27 \%$ sequence identity with $A$. mellifera $\alpha 9$ and $21 \%$ with $A$. gambiae $\alpha 9$, respectively. $\mathrm{Bm} \beta 2$ and $\mathrm{Bm} \beta 3$ show equal sequence identities with $A$. mellifera $\beta 2,17 \%$.

\section{Gene structure}

Figure 3 shows that only subunits $\alpha 6$ of Bombyx and Drosophila share the same exon-intron boundaries. Another pair, subunit Bm $\alpha 8$ and its Drosophila counterpart, $\beta 2$, only differs in one exon boundary. Bma3 and Bma7 possess the same number of exons with their Drosophila counterparts. Bm $\alpha 2$ and $B m \beta 1$ of Bombyx possess more exons than their Drosophila counterparts and $\alpha 1, \alpha 4$, and $\alpha 5$ of Bombyx have fewer exons than their Drosophila counterparts. Surprisingly, three subunits of B. mori, Bma9, $\mathrm{Bm} \beta 2$, and $\mathrm{Bm} \beta 3$, have no introns.

\section{Alternative splicing}

Drosophila nAChR subunit $\alpha 4$ has two alternative forms differing in residues near the Cys-loop; the $\alpha 4$ subunit with exon 4' displays a less efficient assembling ability than that with exon 4 [22]. Silkworm $\alpha 4$ also has these two alternative exons (Figure 4A). Jin et al (2007) cloned $B$. mori $\alpha 6$ and reported that the exons 3 and 8 of this subunit each possess two alternative forms [18]. B. mori $\alpha 6$ could have exon $3 \mathrm{a}$, exon $3 \mathrm{~b}$ or both in tandem. We found a transcript of Bma6 missing both exons $3 \mathrm{a}$ and $3 \mathrm{~b}$ (Figure $4 \mathrm{C}$ ). Alternative splicing of silkworm nAChRs not only occurs in $\alpha 4$ and $\alpha 6$, but also in $\alpha 8$. Silkworm $\alpha 8$ has two alternative exon $7 \mathrm{~s}$, located at TM2 and TM3 (Figure 4A). Two alternative exon $4 \mathrm{~s}$ of $\alpha 4$ are found in Drosophila, Apis, Anopheles, and Bombyx. It has been postulated that these alternative exons have arisen from a very ancient duplication event before the evolutionary divergence of those insects. However, we found that the two alternative exon $7 \mathrm{~s}$ in $\alpha 8$ are extremely similar (86\% identity), indicating a more recent duplication event. A phylogenetic tree was constructed using protein sequences corresponding to the exon $7 \mathrm{~s}$ from several insects, showing that duplication of exon 7s appeared before the divergence of two lepidopteran insects, after the divergence of Apis and Bombyx, and at the same time with the divergence of Bom$b y x$ from two dipteran insects (Figure 4B). 
Table I: Percentage identity/similarity between putative B. mori and D. melanogaster nAChR subunits

\begin{tabular}{|c|c|c|c|c|c|c|c|c|c|c|c|c|}
\hline Subunit & Bmal & $B m \alpha 2$ & $\mathrm{Bm} \alpha 3$ & $\mathrm{Bm} \alpha 4$ & $\mathrm{Bm} \alpha 5$ & Bma6 & Bma7 & Bma8 & $\mathrm{Bm} \beta \mathrm{I}$ & $\mathrm{Bm} \alpha 9$ & $\mathrm{Bm} \beta 2$ & $\mathrm{Bm} \beta 3$ \\
\hline $\mathrm{Bm \alpha l}$ & -- & $53 / 68$ & $54 / 69$ & $48 / 63$ & $25 / 39$ & $33 / 49$ & $33 / 50$ & $54 / 69$ & $37 / 53$ & $15 / 32$ & $13 / 28$ & $11 / 26$ \\
\hline Dmal & $74 / 82$ & $51 / 66$ & $53 / 66$ & $49 / 63$ & $23 / 36$ & $31 / 48$ & $32 / 47$ & $52 / 66$ & $38 / 52$ & $15 / 30$ & $11 / 26$ & $11 / 24$ \\
\hline $\mathrm{Bm} \alpha 2$ & $53 / 68$ & -- & $48 / 65$ & $43 / 60$ & $22 / 36$ & $31 / 50$ & $32 / 50$ & $49 / 66$ & $37 / 54$ & $|5 / 3|$ & $12 / 26$ & $10 / 25$ \\
\hline Dma2 & $50 / 64$ & $77 / 83$ & $48 / 62$ & $42 / 57$ & $22 / 35$ & $30 / 48$ & $32 / 48$ & $47 / 65$ & $36 / 53$ & $|4 / 3|$ & $12 / 26$ & $10 / 25$ \\
\hline $\mathrm{Bm} \alpha 3$ & $54 / 69$ & $48 / 65$ & -- & $62 / 71$ & $26 / 40$ & $34 / 50$ & $36 / 51$ & $55 / 70$ & $40 / 56$ & $18 / 34$ & $11 / 29$ & $13 / 28$ \\
\hline Dma3 & $37 / 47$ & $34 / 45$ & $52 / 56$ & $43 / 51$ & $17 / 26$ & $22 / 35$ & $23 / 34$ & $37 / 47$ & $28 / 38$ & $11 / 22$ & $7 / 19$ & $8 / 18$ \\
\hline $\mathrm{Bm} \alpha 4$ & $48 / 63$ & $43 / 60$ & $62 / 71$ & -- & $22 / 35$ & $31 / 47$ & $30 / 46$ & $49 / 63$ & $36 / 51$ & $15 / 29$ & $10 / 26$ & $11 / 23$ \\
\hline Dma4 & $49 / 62$ & $44 / 60$ & $62 / 71$ & $70 / 77$ & $22 / 36$ & $30 / 47$ & $31 / 48$ & $50 / 64$ & $37 / 53$ & $15 / 29$ & $11 / 26$ & $11 / 23$ \\
\hline $\mathrm{Bm} \alpha 5$ & $25 / 39$ & $22 / 36$ & $26 / 40$ & $22 / 35$ & -- & $27 / 41$ & $28 / 44$ & $24 / 38$ & $24 / 40$ & $16 / 34$ & $14 / 29$ & $11 / 25$ \\
\hline$D m \alpha 5$ & $23 / 34$ & $23 / 34$ & $23 / 33$ & $21 / 32$ & $17 / 26$ & $42 / 49$ & $48 / 53$ & $21 / 34$ & $21 / 33$ & $9 / 20$ & $8 / 18$ & $7 / 17$ \\
\hline $\mathrm{Bm} \alpha 6$ & $33 / 49$ & $31 / 50$ & $34 / 50$ & $31 / 47$ & $27 / 4 \mid$ & -- & $65 / 75$ & $32 / 52$ & $32 / 52$ & $15 / 33$ & $14 / 29$ & $12 / 26$ \\
\hline Dma6 & $32 / 49$ & $32 / 49$ & $34 / 50$ & $31 / 46$ & $28 / 44$ & $79 / 87$ & $66 / 76$ & $33 / 50$ & $32 / 51$ & $16 / 34$ & $14 / 30$ & $12 / 29$ \\
\hline $\mathrm{Bm} \alpha 7$ & $33 / 50$ & $32 / 50$ & $36 / 51$ & $30 / 46$ & $28 / 44$ & $65 / 75$ & -- & $32 / 52$ & $32 / 50$ & $15 / 33$ & $13 / 30$ & $12 / 27$ \\
\hline Dma7 & $32 / 47$ & $32 / 50$ & $34 / 48$ & $29 / 45$ & $25 / 39$ & $60 / 71$ & $70 / 77$ & $31 / 47$ & $29 / 48$ & $13 / 29$ & $11 / 26$ & $10 / 23$ \\
\hline $\mathrm{Bm} \alpha 8$ & $54 / 69$ & $49 / 66$ & $55 / 70$ & $49 / 63$ & $24 / 38$ & $32 / 52$ & $32 / 52$ & -- & $37 / 55$ & $16 / 32$ & $12 / 28$ & $12 / 26$ \\
\hline $\operatorname{Dm} \beta 2$ & $52 / 69$ & $47 / 64$ & $55 / 71$ & $49 / 62$ & $25 / 38$ & $33 / 50$ & $33 / 52$ & $72 / 85$ & $37 / 54$ & $16 / 32$ & $12 / 29$ & $13 / 28$ \\
\hline $\mathrm{Bm} \beta \mathrm{I}$ & $37 / 53$ & $37 / 54$ & $40 / 56$ & $36 / 51$ & $23 / 39$ & $33 / 52$ & $33 / 50$ & $37 / 54$ & -- & $15 / 33$ & $11 / 28$ & $12 / 26$ \\
\hline $\operatorname{Dm} \beta \mathrm{I}$ & $37 / 53$ & $35 / 53$ & $40 / 57$ & $35 / 51$ & $24 / 40$ & $32 / 52$ & $32 / 50$ & $37 / 55$ & $86 / 89$ & $15 / 33$ & $12 / 29$ & $12 / 26$ \\
\hline $\mathrm{Bm} \alpha 9$ & $15 / 32$ & $|5 / 3|$ & $18 / 34$ & $15 / 29$ & $16 / 34$ & $15 / 33$ & $15 / 33$ & $16 / 32$ & $15 / 33$ & -- & $24 / 45$ & $21 / 39$ \\
\hline $\mathrm{Dm} \beta 3$ & $17 / 36$ & $16 / 35$ & $17 / 36$ & $16 / 33$ & $17 / 30$ & $17 / 35$ & $17 / 36$ & $17 / 35$ & $16 / 33$ & $19 / 38$ & $15 / 32$ & $13 / 27$ \\
\hline $\mathrm{Bm} \beta 2$ & $13 / 28$ & $12 / 26$ & $11 / 29$ & $10 / 26$ & $14 / 29$ & $14 / 29$ & $13 / 30$ & $12 / 28$ & $11 / 28$ & $24 / 45$ & -- & $22 / 40$ \\
\hline $\mathrm{Bm} \beta 3$ & $11 / 26$ & $10 / 25$ & $13 / 28$ & $11 / 23$ & $11 / 25$ & $12 / 26$ & $12 / 27$ & $12 / 26$ & $12 / 26$ & $21 / 39$ & $22 / 40$ & -- \\
\hline
\end{tabular}

\section{Truncated transcripts}

Existence of nAChR transcripts lacking some exons or retaining some introns has been reported in many insects as well as in vertebrates $[7,9,23]$. It was supposed that the translation products of these transcripts may function as acetylcholine "sponge" like molluscan ACh-binding protein [24] or change physiological properties by interfering with the function of normal subunits [23]. In our experiments, truncated transcripts of Bma4 and $B m \alpha 5$ were identified (Figure 5). Bma4 $4^{\Delta 6,7,8}$ lacks exons 6-8 which includes Loop F and TM1-3. Bm $\alpha 5^{\Delta 4}$ lacks exon 4 , which introduces a premature stop codon because of a frame shift and only a very short open reading frame is retained. D. melanogaster $\alpha 4$ and $\alpha 5$ also had truncated transcripts, but they lack different exons [7].

\section{RNA editing}

Four $D$. melanogaster $\mathrm{nAChR}$ subunits, $\alpha 5, \alpha 6, \beta 1, \beta 2$, were found having RNA editing sites. Their $B$. mori counterparts, $\alpha 5, \alpha 6, \alpha 8, \beta 1$, were examined for RNA editing sites in our experiments. cDNA clones from separate RT-PCRs and the corresponding regions of genomic DNA were sequenced and compared. While the genomic DNA sequences were same as those in the silkworm genome sequence database [19], five bases of $\alpha 6$ (nts \#392, 394, 395,447 , and 454 ) in the cDNA sequence were different from genome DNA sequence, likely resulting from RNA editing. These nucleotide changes result in altered residues of N131S, N132G, I149M, and T152A. Possible RNA editing was also found in three transcript variants of the $B$. mori $\alpha 6$ gene [18]. No RNA editing sites were found in other three subunit genes examined.

\section{Discussion}

With the help of the silkworm genome sequence and by RT-PCR and RACE, we identified the first nAChR subunit gene family in a lepidopteran insect, and the fourth insect nAChR gene family, following D. melanogaster, A. gambiae, and $A$. mellifera. With 12 members, the nAChR gene family of $B$. mori is the most complex one among those reported for insects. Bm $\alpha 1-8$ and $\beta 1$ are more similar to the counterparts of other insects compared with the other three subunits, $\alpha 9, \beta 2$ and $\beta 3$. The phylogenetic relationship of the three subunits indicated that they might have arisen from two separate evolutionary duplication events. The first event likely took place after Bombyx had diverged from Drosophila and Anopheles, and the second might occur after Bombyx had diverged from Apis.

The GEK motif preceding TM2 is conserved in most insect nAChR subunits, and the glutamate residue is considered to be associated with cation selectivity [2]. Sequence comparison results show that most of the $B$. mori nAChR subunits contained a GEK motif preceding TM2 (Figure 1), while the motif was replaced by STR in $\alpha 9$ and $\beta 3$, and by TIR in $\beta 2$. We calculated the amino acid identities of these three subunits with anion channel subunits including $D$. melanogaster GABAR, RDL [GenBank: NM 079267], glutamate-gated chloride channel [GenBank: U158776], histamate-gated chloride channel [GenBank: AF382401], and vertebrate serotonin receptor [GenBank: AJ005205]. B. 


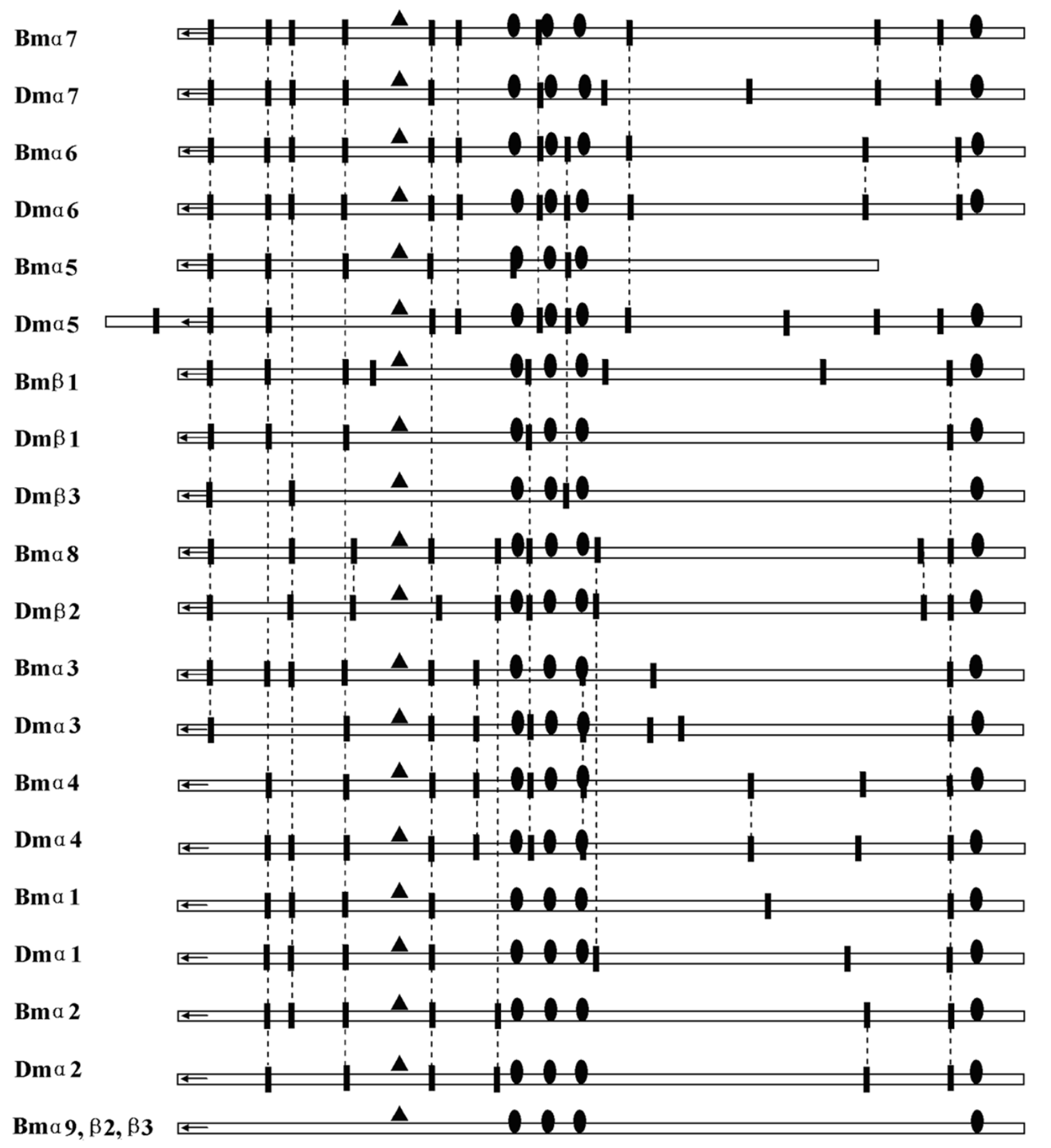

Figure 3

Comparison of exon junctions of Bombyx mori and Drosophila melonogaster nAChRs. N-terminal signal leader peptides are symbolized by arrows. Exon junctions are indicated by vertical bars. Sites of transmembrane motifs are indicated by ovals and Cys-loops are showed by triangles. Identical exon boundaries are showed by dotted lines. 
$\mathbf{A}$

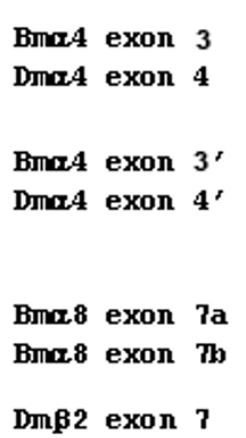

B

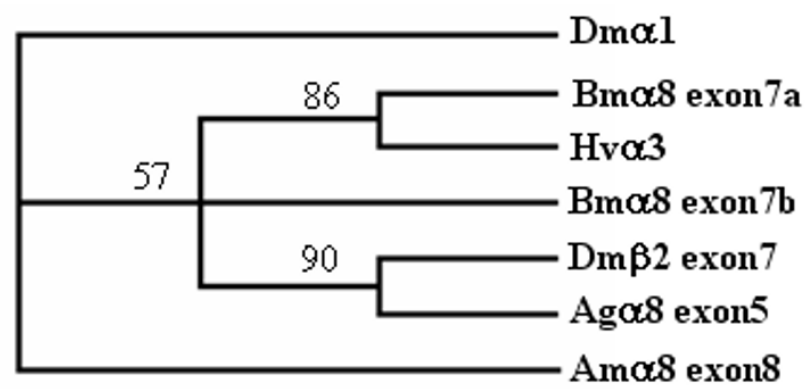

\begin{abstract}
------LpE-------
$\star$

* $\quad-$-LpB-

ADGAFEY TL AT TKA TIYHQGLVEYKP PAIYKS SCE DVEYF PFDEQT CVLKFGSWTYDGFK ADGNEEV TLATKATTY $\overline{S E G L V E W K P ~ P A I Y K S ~ S C E ~ I D V E Y F ~ P F D E Q T ~ C V L K F G S W T Y D G F K ~}$
\end{abstract}

ADGICEV TLMTKA TUYYYIGLVYWP PAVYKS SCS IDVEFF PYDYQT CVLKLGSWTYDGFK

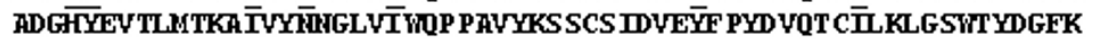

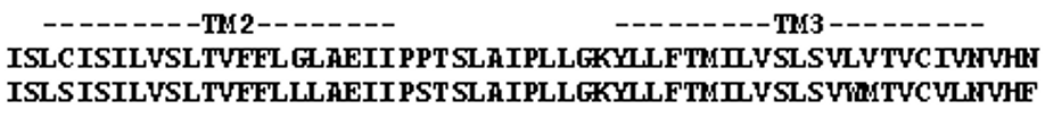

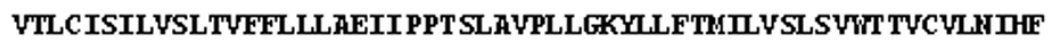

Figure 4

Alternative splicing of Bombyx mori nAChRs. A) Comparison of corresponding exons of Bombyx and Drosophila nAChRs. LpE and LpB indicated are loops involved in acetylcholine binding. Two asterisks stand for Cys residues forming disulfide bond separated by 13 amino acid residues. TM2 and TM3 are transmembrane motifs engaged in forming the cation permeable channel. B) Phylogenetic relationship of Bma8 exon 7s and their corresponding exons from Heliothis, Apis, Anopheles and Drosophila. Protein sequence of $\mathrm{Dm \alpha}$ I corresponding to $\mathrm{Bm \alpha 8}$ exon $7 \mathrm{~s}$ is included as an outgroup. Numbers at each node represent bootstrap values with I,000 replicates. C) Four splicing patterns between exons 2 and 4 of Bombyx mori nAChR $\alpha 6$. mori $\alpha 9$ shares $10-17 \%$ identities with those anion channel subunits, while it shares $27 \%$ identity with Apis nAChR $\alpha 9$. Bombyx $\beta 2, \beta 3$ share $8-12 \%$ identities with above anion channel subunits, they show $17 \%$ identities with A. mellifera $\beta 2$. These results show that Bombyx $\alpha 9, \beta 2$ and $\beta 3$ are likely $n A C h R$ subunit candidates as opposed to being other members of the cys-loop LGIC superfamily. Whether Bombyx $\alpha 9, \beta 2$ and $\beta 3$ possess the cation selectivity as other GEK-motif-containing AChR subunits remains to be determined.

It is an unusual finding that $B$. mori $\alpha 5$ only has three transmembrane regions, lacking TM4. Our 3' RACE result showed the $\alpha 5$ mRNA contained a consensus polyadenylation signal (AATAAA) located 22 bp upstream of the poly(A) tail. According to the 3'-terminal sequence, we designed gene specific primers and performed additional RT-PCR, but failed to find any longer cDNA product that would include TM4 or another distinct region. These results suggest that $B$. mori $\alpha 5$ contains only three transmembrane regions.

Alternative splicing of several exons results in changes of residues belonging to Loops D, E, Cys-loop, TM2, and TM3. The truncated transcripts lack exons that contain sequences for ligand binding or the ion channel pore. We also detected possible RNA editing events in $\mathrm{nAChR}$ genes. It was reported that RNA editing might change during insect development [18], so additional editing sites might exist in $\alpha 6$ or other subunits.

\section{Conclusion}

Our results show the silkworm contains the largest insect nAChR gene family characterized to date, including nine $\alpha$-type subunits and three $\beta$-type subunits. RNA-editing, alternative splicing and truncated transcripts were found in several subunit genes, which might further enhance the diversity of the gene family. These results provide a foun- 


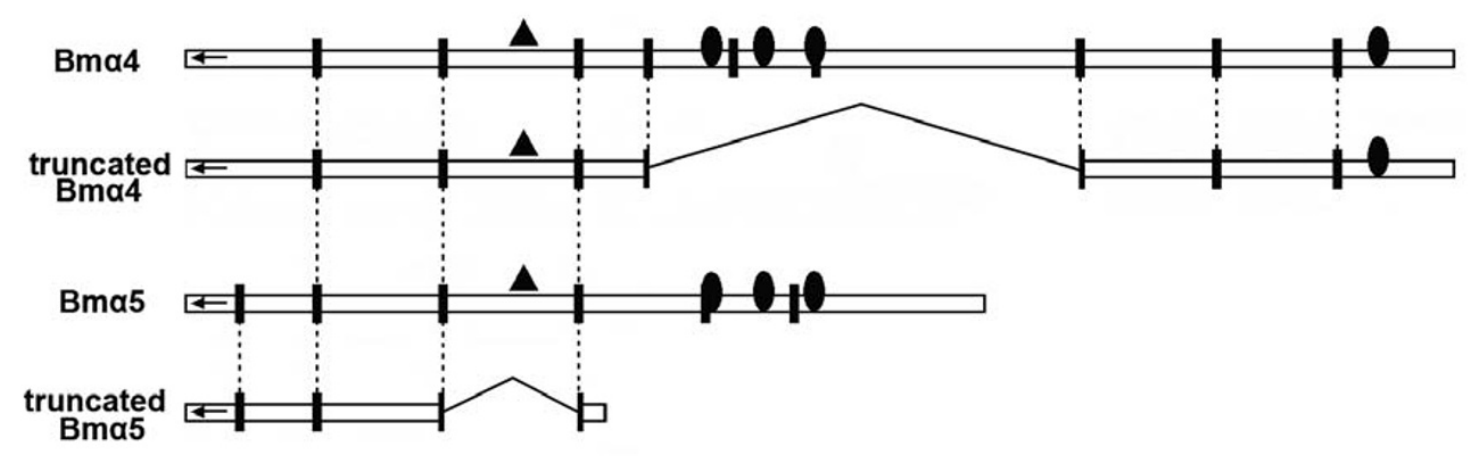

\section{Figure 5}

Comparison of $\mathrm{Bm} \alpha 4, \mathrm{Bm} \alpha \mathbf{5}$, and their truncated transcripts. Missed exons are displaced by bridge lines. $\mathrm{N}$-terminal signal leader peptides are symbolized by arrows. Exon junctions are indicated by vertical bars. Sites of transmembrane motif are indicated by ovals and Cys-loops are showed by triangles. Identical exon boundaries are showed by dotted lines. A short bar downstream of the bridge line of truncated Bm 15 indicates a premature stop codon because of a frame shift.

dation for future characterization of possibly tissue-specific and developmental expression patterns and the physiological functions of lepidopteran nAChR receptors. Furthermore, the comparison of the nAChR gene family of Bombyx with those of other insects, in particular Lepidoptera, may serve as an important basis for developing improved insecticides that spare an economically important insect while controlling major agricultural pests.

\section{Methods}

Insects

The fertilized B. mori (Strain Dazao) parent eggs were kindly presented by Dr. Li Muwang in the Silkworm Research Institute, Agricultural Academy of China, and the larvae were reared with artificial diet at $25^{\circ} \mathrm{C}$.

\section{Bioinformatics analysis and identification of $\mathrm{nAChR}$ subunits in the Bombyx genome}

The Bombyx mori genome sequence database [19] was examined with all D. melanogaster and A. mellifera nAChR sequences using the tBlastn algorithm [25] to produce candidate gene fragments. For each subunit, the gene sequences having the highest similarity were used to design gene specific primers for RT-PCR [see Additional file 1] and RACE [see Additional file 2]. 5' of Bma7 was obtained by searching the EST database using Blastn method [20].

\section{RT-PCR}

Total RNA was extracted from heads of 4 th instar larvae using TRIzol reagent (Dingguo, Shanghai, China) according to the protocol. One $\mu \mathrm{g}$ total RNA was used for synthesizing the first-strand cDNA using PrimeScript ${ }^{\mathrm{TM}}$ Reverse Transcriptase (Takara, Dalian, China) and gene specific primers. Subunit-specific oliogonucleotide primer pairs annealing to different exons were designed to generate genomic and cDNA PCR products that differed in size (except for three subunit genes without any intron). Following PCR reactions were performed in a total volume of $25 \mu \mathrm{L}$ composed of $1 \times$ PCR buffer, $0.2 \mathrm{mM} \mathrm{dNTP} \mathrm{mix,} 1.5$ $\mathrm{mM} \mathrm{MgCl}{ }_{2}, 1 \mathrm{U}$ Taq polymerase (Takara, Dalian, China), $0.4 \mu \mathrm{M}$ each primer, and $1 \mu \mathrm{L}$ cDNA template.

Some N-terminal signal peptides and C-termini are highly variable between subunits, and could not be identified with confidence by BLAST. In these cases, 5'- and 3'-RACE were performed using the SMART ${ }^{\mathrm{TM}}$ RACE cDNA Amplification Kit (Clontech, USA) to obtain the complete ORF sequences. For 5'-RACE, the cDNA was synthesized using gene specific primers. For both 3'-RACE and 5'-RACE, following PCR amplification we used another round of nested PCR. Other PCR conditions refer to the manual.

All PCR products were analyzed by electrophoresis in $1 \%$ agarose gel, and DNA fragments were purified with the DNA Fragment quick purification/recovery kit (Dinguo, Shanghai, China). The purified DNA fragments were ligated into pMD 18-T (Takara, Dalian, China), and sequenced with an ABI-3730 automatic sequencer. The sequences reported in this paper have been deposited in GenBank with accession numbers: Bm $\beta 1$ [GenBank: EU082071]; Bm $\beta 2$ [GenBank: EU082072]; Bm $\beta 3$ [Gen-

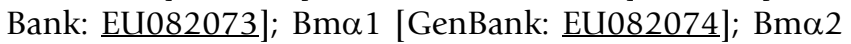
[GenBank: EU082075]; Bma3 [GenBank: EU082076]; Bmo4 variant 1 [GenBank: EU082077]; Bmo4 variant 2 [GenBank: EU082078]; Bma4 variant 3 [GenBank: EU082079]; Bma5 variant 1 [GenBank: EU082080]; Bmo5 variant 2 [GenBank: EU082081] ; Bma6 variant 2 
[GenBank: EU082082]; Bma6 variant 4 [GenBank: EU082083]; Bma7 [GenBank: EU082084]; Bma8 variant 1 [GenBank: EU082085]; Bma8 variant 2 [GenBank: EU082086]; Bma9 [GenBank: EU082087].

\section{Sequence and phylogenetic analyses}

Signal peptide cleavage sites were predicted using the SignalP 3.0 server program [26]. Transmembrane regions were speculated using the TMpred program [27]. Potential post-translational modification sites including those for $\mathrm{N}$-glycosylation, cyclic AMP (cAMP)-binding, and protein kinase $\mathrm{C}$ (PKC), CK2, and tyrosine kinase phosphorylation were identified using the PROSITE database [28]. All B. mori nAChR subunit protein sequences were aligned using software ClustalX [29] and edited with software GeneDoc [30]. Similarity and identity values between nAChR sequences were the output from the GeneDoc program. All Drosophila, Apis, Anopheles, and partial lepidopteran $\mathrm{nAChR}$ subunit protein sequences were aligned with ClustalX and the multiple alignment result was used as input into the PAUP software (v.4.0b10) to construct phylogenetic trees with the maximum parsimony method and bootstrapping sampled for 1,000 times [31], and the tree was viewed with TreeView application [32].

\section{Authors' contributions}

YMS carried out the molecular cloning and analysis of the sequences and drafted the manuscript. KD helped to design the study and draft the manuscript. CXZ conceived of the study, and participated in its design and coordination and helped to draft the manuscript. All authors read and approved the final manuscript.

\section{Additional material}

\section{Additional file 1}

Sequences of RT-PCR primers used in this study. All sequences are showed in $5^{\prime} \rightarrow 3^{\prime}$ direction.

Click here for file

[http://www.biomedcentral.com/content/supplementary/14712164-8-324-S1.pdf]

\section{Additional file 2}

Sequences of RACE primers used in this study. One gene specific primer and one universal primer are used in each round of PCR. Only gene specific primers are listed and universal primers are UPM in first round PCR and nested NUP in second round PCR according to the user manual. All sequences are showed in $5^{\prime} \rightarrow 3^{\prime}$ direction.

Click here for file

[http://www.biomedcentral.com/content/supplementary/14712164-8-324-S2.pdf]

\section{Acknowledgements}

We thank Dr. Li Muwang in the Silkworm Research Institute, Agricultural Academy of China, and Professor Lu Xingmeng in the Department of Seri- culture, Zhejiang University, for providing the eggs or larvae of the silkworm. This project was supported by the National Program of High-tech Research and Development (863 High-Tech Program, No.

2006AA IOA I 19), the National Natural Science Foundation of China (Project No. 30770280) and the National Basic Research Program of China (2003CB I 14403).

\section{References}

I. Lee D, O'Dowd DK: Fast excitatory synaptic transmission mediated by nicotinic acetylcholine receptors in Drosophila neurons. J Neurosci 1999, I 9( I3):53 I I-532I.

2. Corringer PJ, Le Novere N, Changeux JP: Nicotinic receptors at the amino acid level. Annu Rev Pharmacol Toxicol 2000, 40:43 I-458.

3. Ihara M, Matsuda K, Otake M, Kuwamura M, Shimomura M, Komai K, Akamatsu M, Raymond V, Sattelle DB: Diverse actions of neonicotinoids on chicken $\alpha 7, \alpha 4 \beta 2$ and Drosophila-chicken SAD $\beta 2$ and ALS $\beta 2$ hybrid nicotinic acetylcholine receptors expressed in Xenopus laevis oocytes. Neuropharmacology 2003, 45(I): I33-I 44.

4. Kao PN, Karlin A: Acetylcholine receptor binding site contains a disulfide cross-link between adjacent half-cystinyl residues. J Biol Chem 1986, 26 I ( 18):8085-8088.

5. Hopfield JF, Tank DW, Greengard P, Huganir RL: Functional modulation of the nicotinic acetylcholine receptor by tyrosine phosphorylation. Nature 1988, 336(6200):677-680.

6. Thany SH, Lenaers G, Raymond-Delpech V, Sattelle DB, Lapied B: Exploring the pharmacological properties of insect nicotinic acetylcholine receptors. Trends Pharmacol Sci 2007, 28(I): 14-22.

7. Sattelle DB, Jones AK, Sattelle BM, Matsuda K, Reenan R, Biggin PC: Edit, cut and paste in the nicotinic acetylcholine receptor gene family of Drosophila melanogaster. Bioessays 2005, 27(4):366-376.

8. Jones AK, Grauso M, Sattelle DB: The nicotinic acetylcholine receptor gene family of the malaria mosquito, Anopheles gambiae. Genomics 2005, 85(2): 176-187.

9. Jones AK, Raymond-Delpech V, Thany SH, Gauthier M, Sattelle DB: The nicotinic acetylcholine receptor gene family of the honey bee, Apis mellifera. Genome Res 2006, I6(I I): | 422-I 430.

10. Millar NS: Assembly and subunit diversity of nicotinic acetylcholine receptors. Biochem Soc Trans 2003, 3 I (Pt 4):869-874.

II. Millar NS, Denholm I: Nicotinic acetylcholine receptors: targets for commercially important insecticides. Invert Neurosci 2007, 7(I):53-66.

12. Matsuda K, Buckingham SD, Kleier D, Rauh JJ, Grauso M, Sattelle DB: Neonicotinoids: insecticides acting on insect nicotinic acetylcholine receptors. Trends Pharmacol Sci 200I, 22(I I):573-580.

13. Tomizawa M, Casida JE: Neonicotinoid insecticide toxicology: mechanisms of selective action. Annu Rev Pharmacol Toxicol 2005 , 45:247-268

14. Smith GE, Summers MD, Fraser MJ: Production of human beta interferon in insect cells infected with a baculovirus expression vector. Mol Cell Biol I983, 3(I 2):2I56-2I65.

15. Goldsmith MR, Wilkins AS: Molecular model systems in the Lepidoptera. Cambridge, Cambridge University Press; 1995.

16. Mita K, Kasahara M, Sasaki S, Nagayasu Y, Yamada T, Kanamori H, Namiki N, Kitagawa M, Yamashita H, Yasukochi Y, Kadono-Okuda $K$, Yamamoto K, Ajimura M, Ravikumar G, Shimomura M, Nagamura Y, Shin IT, Abe H, Shimada T, Morishita S, Sasaki T: The genome sequence of silkworm, Bombyx mori. DNA Res 2004, II (I):27-35.

17. Xia Q, Zhou Z, Lu C, Cheng D, Dai F, Li B, Zhao P, Zha X, Cheng T, Chai C, Pan G, Xu J, Liu C, Lin Y, Qian J, Hou Y, Wu Z, Li G, Pan M, Li C, Shen Y, Lan X, Yuan L, Li T, Xu H, Yang G, Wan Y, Zhu Y, Yu M, Shen W, Wu D, Xiang Z, Yu J, Wang J, Li R, Shi J, Li H, Li G, Su J, Wang X, Li G, Zhang Z, Wu Q, Li J, Zhang Q, Wei N, Xu J, Sun H, Dong L, Liu D, Zhao S, Zhao X, Meng Q, Lan F, Huang X, Li Y, Fang $L$, Li C, Li D, Sun Y, Zhang Z, Yang Z, Huang Y, Xi Y, Qi Q, He D, Huang $\mathrm{H}$, Zhang X, Wang Z, Li W, Cao Y, Yu Y, Yu H, Li J, Ye J, Chen $\mathrm{H}$, Zhou Y, Liu B, Wang J, Ye J, Ji H, Li S, Ni P, Zhang J, Zhang Y, Zheng H, Mao B, Wang W, Ye C, Li S, Wang J, Wong GK, Yang H: A draft sequence for the genome of the domesticated silkworm (Bombyx mori). Science 2004, 306(5703): 1937-1940.

18. Jin $Y$, Tian N, Cao J, Liang J, Yang Z, Lv J: RNA editing and alternative splicing of the insect $n A C h R$ subunit alpha6 tran- 
script: evolutionary conservation, divergence and regulation. BMC Evol Biol 2007, 7:98.

19. BLAST with arthropoda genomes [http://
[ www.ncbi.nlm.nih.gov/sutils/genom table.cgi?organism=insects]

20. NCBI BLAST [http://www.ncbi.nlm.nih.gov/BLAST/]

21. Nishizaki T: N-glycosylation sites on the nicotinic $A C h$ receptor subunits regulate receptor channel desensitization and conductance. Brain research 2003, II4(2): 172-176.

22. Lansdell SJ, Millar NS: Cloning and heterologous expression of $\mathrm{D} \alpha 4$, a Drosophila neuronal nicotinic acetylcholine receptor subunit: identification of an alternative exon influencing the efficiency of subunit assembly. Neuropharmacology 2000, 39(13):2604-2614.

23. Saragoza PA, Modir JG, Goel N, French KL, Li L, Nowak MW, Stitzel JA: Identification of an alternatively processed nicotinic receptor $\alpha \mathbf{7}$ subunit RNA in mouse brain. Brain research 2003, II (7): $15-26$.

24. Smit AB, Brejc K, Syed N, Sixma TK: Structure and function of $A C h B P$, homologue of the ligand-binding domain of the nicotinic acetylcholine receptor. Ann N Y Acad Sci 2003, 998:81-92.

25. Altschul SF, Gish W, Miller W, Myers EW, Lipman DJ: Basic local alignment search tool. J Mol Biol 1990, 215(3):403-4I0.

26. SignalP 3.0 Server [http://www.cbs.dtu.dk/services/SignalP/]

27. TMpred - Prediction of Transmembrane Regions and Orientation [http://www.ch.embnet.org/software/TMPRED form.html]

28. PROSITE SCAN [http://npsa-pbil.ibcp.fr/cgi-bin/ npsa automat.pl!page $=$ npsa prosite.html]

29. Thompson JD, Gibson TJ, Plewniak F, Jeanmougin F, Higgins DG: The CLUSTAL_X windows interface: flexible strategies for multiple sequence alignment aided by quality analysis tools. Nucleic Acids Res 1997, 25(24):4876-4882.

30. Nicholas KB, Nicholas HB], Deerfield DW: GeneDoc: Analysis and Visualization of Genetic Variation. EMBNEW NEWS 1997, 4: 14.

31. Felsenstein J: Confidence limits on phylogenies: an approach using the bootstrap. Evolution 1985, 39(4):783-791.

32. Page RD: TreeView: an application to display phylogenetic trees on personal computers. Comput Appl Biosci 1996, I2(4):357-358.

Publish with Bio Med Central and every scientist can read your work free of charge

"BioMed Central will be the most significant development for disseminating the results of biomedical research in our lifetime. "

Sir Paul Nurse, Cancer Research UK

Your research papers will be:

- available free of charge to the entire biomedical community

- peer reviewed and published immediately upon acceptance

- cited in PubMed and archived on PubMed Central

- yours - you keep the copyright

Submit your manuscript here:

http://www.biomedcentral.com/info/publishing_adv.asp
BioMedcentral 